\title{
Freezing of Moduli with Fluxes in Three Dimensions
}

\author{
Riccardo Argurio, Vanicson L. Campos, \\ Gabriele Ferretti and Rainer Heise \\ Institute for Theoretical Physics - Göteborg University and \\ Chalmers University of Technology, 41296 Göteborg, Sweden
}

\begin{abstract}
We study warped compactifications to three dimensions, realized as an orientifold of type IIA string theory on $T^{7}$. By turning on 3and 4-form fluxes on the torus in a supersymmetric way, we generate a potential for the moduli fields. We present various flux configurations with $\mathcal{N}=1,2,3,4,5,6$ supersymmetries and count the number of moduli in each case. In particular, we show that there are $\mathcal{N}=1$ configurations where all but one of the moduli are frozen.
\end{abstract}




\section{Introduction}

Generic supersymmetric compactifications of string and M-theory lead to a low-energy effective theory in the non-compact dimensions which contains a number of moduli fields. These are massless scalars whose expectation value at infinity parameterizes the vacua of the low-energy theory. Typically, it would be interesting to find compactifications which lead to no moduli, for two main reasons. One is that in our four dimensional world (or its minimal supersymmetric extension) we do not have any massless scalar field. The other reason is that a compactification without moduli would give us a direct relation between string theory and low-energy parameters, that is no tuning of the compactification to fit low-energy data. This is of course desirable if the ultimate aim is to derive low-energy physics entirely from first principles.

Of course, finding a string vacuum with no moduli is not an easy task. Recently, an interesting avenue for obtaining compactifications with a reduced number of moduli has been considered. It consists in turning on fluxes of the form field strengths along the compactification manifold [1] 22] (see 23] for a different approach based on asymmetric orbifolds yielding very few moduli). Generically, one can give a number of conditions on the fluxes which imply that the configuration preserves a certain amount of supersymmetry in the non-compact dimensions. Additionally, these supersymmetric fluxes contribute to a tadpole cancellation condition, so that either manifolds with non-trivial topology or orientifolds have to be considered. Another interesting feature of these models is that they provide a warp factor which implies that the scale in the non-compact dimensions depends on the position in the compact manifold. This can potentially lead to hierarchies in the low-energy theory (see e.g. [8, 9, 10, 15]).

If one considers a vacuum where supersymmetry is totally broken, then most moduli will generically be massive at tree level, and the others are likely to acquire masses by loop corrections. However, there is also the danger that some of them become actually tachyonic or have a potential unbounded from below. We thus prefer to consider supersymmetric configurations where these problems do not arise.

In [18], a simple though illuminating example of moduli stabilization through fluxes is considered. It consists of an orientifold of type IIB theory on $T^{6}$, with 3 -form fluxes turned on to compensate (at least partially) for the O3-charge tadpole, while keeping $\mathcal{N}=1$ supersymmetry in 4 dimensions. It is shown that in the "best" case scenario, when all the O3-charge is compensated by fluxes (the addition of any D3-brane brings in the moduli

relative to its position), one is left with 3 complex moduli, including most 
notably the volume of the torus. Though there remain some moduli, it is still a drastic reduction, since one started from the 19 complex closed string moduli together with the 48 complex moduli corresponding to the position of the 16 D3-branes present in the configuration without fluxes.

In this note, we consider a 3 dimensional orientifold model. By going to a lower dimensional theory, we have the possibility to consider a configuration with only 2 supercharges, that is $\mathcal{N}=1$ in 3 dimensions. Lower supersymmetry implies less constraints on the low-energy Lagrangian, so that we expected to have fewer moduli than in the case considered in [18]. In particular, we find models in which all but one of the moduli are frozen.

Our model is built as follows. We consider an orientifold of type IIA theory on $T^{7}$. In order to cancel the tadpole generated by the O2-planes, we turn on the most general configuration of fluxes which preserves at least the minimal amount of supersymmetry in 3 dimensions. If the fluxes alone cancel the O2-charge, the only potential moduli present are the ones arising from closed strings. Without fluxes there are 64 of them. Some of them actually arise as massless abelian vectors, but in 3 dimensions these are Hodge dual to massless scalars. We will show that turning on the fluxes, many of the moduli (in the best case all but one) are frozen. More specifically, the metric moduli and the other scalars are fixed by the condition for the fluxes to be supersymmetric. However, a combination of the dilaton and the volume of the torus is always a free modulus. The remaining 28 moduli would arise by dualizing the massless vectors in the NSNS and RR sectors. However most of them (in the best case all of them) acquire a mass through the Chern-Simons terms present in their equations of motion, and thus cannot be dualized. An interesting consequence of this effect is that in the generic case the topological mass matrix for these fields is such that the theory breaks parity.

The outline of the paper is as follows. In section 2 we present the orientifold and derive the fields which are present after the projection, including the allowed fluxes. In section 3 we consider the action of type IIA supergravity, and how the requirement of preserving supersymmetry in three dimensions leads to constraints on the fluxes that can be turned on. In section 4 we consider the tadpole cancellation and the Dirac quantization condition, and show how the moduli are frozen, giving a number of explicit examples, including an $\mathcal{N}=1$ example where all the moduli but one acquire a mass. In section 5 we discuss the 11 dimensional alternative interpretation of our construction. We conclude with some additional discussions. 


\section{Type IIA on a $T^{7} / \mathbf{Z}_{2}$ orientifold}

Let us begin by reviewing the construction of type IIA string theory on a $T^{7} / \mathbf{Z}_{2}$ orientifold.

Contrary to type IIB string theory, where world-sheet parity $\Omega$ and space-time left fermion number $(-1)^{F_{L}}$ are separately symmetries of the theory, in type IIA, only $(-1)^{F_{L}}$ is a symmetry because $\Omega$ would map spinors with opposite chirality into each other. However, $\Omega$ can be combined with a parity transformation, that is the reflection of an odd number of space coordinates, to yield a symmetry because parity is implemented on the fermions by an odd number of gamma matrices. We shall consider the generator of the $\mathbf{Z}_{2}$ orientifold group to be $\Omega I_{7}$, where $I_{7}$ is the reflection of the seven coordinates of the torus. Our notation will be $M=(\mu, i)$ where $\mu=0,1,2$ are the uncompactified coordinates of three dimensional Minkowski space-time and $i=3, \cdots, 9$ the coordinates on the torus.

We will be using a mostly plus metric for which it is possible to choose the ten-dimensional gamma matrices $\Gamma^{M}$ to be real $\left(\Gamma^{0 T}=-\Gamma^{0}\right)$ and define the action of $\Omega I_{7}$ on the right- and left-moving spin-fields $S_{ \pm}$:

$$
\Omega I_{7}: S_{ \pm} \rightarrow P S_{\mp},
$$

where the index \pm denotes the ten-dimensional chirality: $\Gamma^{11} S_{ \pm}= \pm S_{ \pm}$. Of the two possible choices for the $P$-matrix $\left(P=\Gamma^{012}\right.$ and $P=\Gamma^{012} \Gamma^{11} \equiv$ $\left.\Gamma^{3456789}\right)$ we choose the first because it squares to +1 thus ensuring that $\left(\Omega I_{7}\right)^{2}=1$ on the supercharges. Equivalently, we could have chosen the second one and compensated for the minus sign by the addition of $(-1)^{F_{L}}$.

We can now study the "parity" of the various massless bosonic fields by computing how they transform under $\Omega I_{7}$. For the NSNS fields $\Phi, G_{M N}$ and $B_{M N}$ the analysis is the same as in the case of the IIB where:

$$
\begin{aligned}
\Omega I_{7}: \Phi & \rightarrow \Phi \\
G_{\mu \nu} & \rightarrow G_{\mu \nu} \\
G_{\mu i} & \rightarrow-G_{\mu i} \\
G_{i j} & \rightarrow G_{i j} \\
B_{\mu \nu} & \rightarrow-B_{\mu \nu} \\
B_{\mu i} & \rightarrow B_{\mu i} \\
B_{i j} & \rightarrow-B_{i j} .
\end{aligned}
$$

For the RR fields $A_{M}$ and $A_{M N P}$ we use the action of (1) on the spin fields $S_{ \pm}$entering the definition of the vertex operators and the following relation, 
(valid for any pair of ten-dimensional Majorana spinors $\epsilon$ and $\lambda$ ):

$$
\bar{\epsilon} \Gamma^{M_{1} \cdots M_{n}} \lambda=(-1)^{n} \bar{\lambda} \Gamma^{M_{n} \cdots M_{1}} \epsilon
$$

to get

$$
\begin{aligned}
\Omega I_{7}: A_{\mu} & \rightarrow-A_{\mu} \\
A_{i} & \rightarrow A_{i} \\
A_{\mu \nu \rho} & \rightarrow A_{\mu \nu \rho} \\
A_{\mu \nu i} & \rightarrow-A_{\mu \nu i} \\
A_{\mu i j} & \rightarrow A_{\mu i j} \\
A_{i j k} & \rightarrow-A_{i j k} .
\end{aligned}
$$

As an example, consider the vertex operator for $F_{\mu i}=\partial_{\mu} A_{i}-\partial_{i} A_{\mu}$ : $V \propto \bar{S}_{-} \Gamma^{\mu i} S_{+}$. Under the action of $\Omega I_{7}$ :

$$
V \rightarrow S_{+}^{T} P^{T} \Gamma^{0} \Gamma^{\mu i} P S_{-}=-\bar{S}_{+} \Gamma^{\mu i} S_{-}=-\bar{S}_{-} \Gamma^{i \mu} S_{+}=V .
$$

Thus, $\Omega I_{7}: F_{\mu i} \rightarrow F_{\mu i}$. The various transformation properties for the gauge potentials in (4) follow from these types of calculation taking into account the extra minus sign coming from $\partial_{i}$ when going from $F$ to $A$.

We see that $F_{i j k l}=4 \partial_{[i} A_{j k l]}$ and $H_{i j k}=3 \partial_{[i} B_{j k]}$ have the right parity properties to be constant fluxes but not $F_{i j}=2 \partial_{[i} A_{j]}$.

Now recall that, in three dimensions, the metric and a two-form or threeform gauge potential carry no degree of freedom, whereas a massless vector field can be dualized into a scalar. When counting the total number of scalars that are not projected out, we must count also those that come from dualizing a vector. The complete list is thus

$$
\begin{aligned}
\Phi & : 1 \text { scalar } \\
G_{i j} & : 28 \text { scalars } \\
B_{\mu i} & : 7 \text { vectors } \\
A_{i} & : 7 \text { scalars } \\
A_{\mu i j} & : 21 \text { vectors }
\end{aligned}
$$

for a total of 64 bosonic degrees of freedom. 


\section{Type IIA dynamics}

The bosonic part of the action of type IIA supergravity, written in the Einstein frame $\left(G_{M N}^{\text {Einst }}=e^{-\phi / 2} G_{M N}^{\text {string }}\right)$, is the following [24]:

$$
\begin{aligned}
S=\frac{1}{(2 \pi)^{7} \alpha^{4}} \int d^{10} x & \left\{\sqrt { - G } \left[R-\frac{1}{2} \partial_{M} \phi \partial^{M} \phi-\frac{1}{12} e^{-\phi} H_{M N P} H^{M N P}\right.\right. \\
& \left.-\frac{1}{4} e^{\frac{3 \phi}{2}} F_{M N} F^{M N}-\frac{1}{48} e^{\frac{\phi}{2}} \tilde{F}_{M N P Q} \tilde{F}^{M N P Q}\right] \\
& \left.-\frac{1}{(48)^{2}} \varepsilon^{M N P Q R S T U V W} B_{M N} F_{P Q R S} F_{T U V W}\right\} .
\end{aligned}
$$

In the above equation we have defined:

$$
\tilde{F}_{M N P Q}=F_{M N P Q}+4 A_{[M} H_{N P Q]} .
$$

All the field strengths are defined as usual as $H_{M N P}=3 \partial_{[M} B_{N P]}$ and similarly for the others, and we take the tensor $\varepsilon^{M N P Q R S T U V W}$ to be independent of the metric $G_{M N}$, with $\varepsilon^{0123456789}=-1$.

The supersymmetry transformations of type IIA supergravity in a purely bosonic background are given by the dilatino and gravitino variations as follows [24]:

$$
\begin{aligned}
\delta \lambda= & -D_{M} \phi \Gamma^{M} \Gamma^{11} \epsilon+\frac{3}{8} e^{\frac{3 \phi}{4}} \Gamma^{M N} F_{M N} \epsilon \\
+ & \frac{1}{12} e^{-\frac{\phi}{2}} \Gamma^{M N L} H_{M N L} \epsilon+\frac{1}{96} e^{\frac{\phi}{4}} \Gamma^{M N L P} \tilde{F}_{M N L P} \Gamma^{11} \epsilon \\
\delta \Psi_{M}= & D_{M} \epsilon-\frac{1}{64} e^{\frac{3 \phi}{4}}\left(\Gamma_{M}{ }^{N L}-14 \delta_{M}^{N} \Gamma^{L}\right) F_{N L} \Gamma^{11} \epsilon \\
& +\frac{1}{96} e^{-\frac{\phi}{2}}\left(\Gamma_{M}{ }^{N L P}-9 \delta_{M}^{N} \Gamma^{L P}\right) H_{N L P} \Gamma^{11} \epsilon \\
& +\frac{1}{256} e^{\frac{\phi}{4}}\left(\Gamma_{M}{ }^{N L P Q}-\frac{20}{3} \delta_{M}^{N} \Gamma^{L P Q}\right) \tilde{F}_{N L P Q} \epsilon
\end{aligned}
$$

where $\left\{\Gamma^{M}, \Gamma^{N}\right\}=2 G^{M N}, \Gamma^{11}$ is independent of the metric and the supersymmetry parameter $\epsilon$ is a real Majorana 32-component spinor.

To solve the above supersymmetry equations we take a D2-brane-like ansatz in terms of the warp factor $\Delta$ as follows:

$$
\begin{aligned}
& d s^{2}=g_{\mathrm{s}}^{-1 / 2}\left(\Delta^{-\frac{5}{8}} \eta_{\mu \nu} d x^{\mu} d x^{\nu}+\Delta^{\frac{3}{8}} g_{m n} d x^{m} d x^{n}\right) \\
& F_{(4)}=g_{\mathrm{s}}^{-1} d x^{0} \wedge d x^{1} \wedge d x^{2} \wedge d \Delta \Delta^{-2}, \quad e^{\phi}=g_{\mathrm{s}} \Delta^{\frac{1}{4}} .
\end{aligned}
$$


We also allow for non-zero fluxes $H_{i j k}$ and $F_{i j k l}$ and take the supersymmetry parameter to satisfy $\Gamma^{012} \epsilon=\epsilon$ (where here the indices are flat).

Then the vanishing of the dilatino equation (9) implies the relation:

$$
\frac{1}{12} e^{-\frac{\phi}{2}} \Gamma^{m n l} H_{m n l} \epsilon+\frac{1}{96} e^{\frac{\phi}{4}} \Gamma^{m n l p} \tilde{F}_{m n l p} \Gamma^{11} \epsilon=0 .
$$

The vanishing of the gravitino variation (10) along the $\mu$-direction implies instead (assuming, of course, $\partial_{\mu} \epsilon=0$ ):

$$
\frac{1}{96} e^{-\frac{\phi}{2}} \Gamma_{\mu}^{m n p} H_{m n p} \Gamma^{11} \epsilon+\frac{1}{256} e^{\frac{\phi}{4}} \Gamma_{\mu}^{m n l p} \tilde{F}_{m n l p} \epsilon=0 .
$$

Equations (12) and (13) together with $\Gamma^{012} \epsilon=\epsilon$ require:

$$
\begin{aligned}
& H \epsilon=0, \\
& \tilde{H} \epsilon=0,
\end{aligned}
$$

where the slash represents the contraction of the fluxes with the gamma matrices.

Finally, we are left with the $\delta \Psi_{m}=0$ condition, which, after a rescaling of the spinor $\epsilon \rightarrow \Delta^{-5 / 32} \epsilon$ gives:

$\frac{1}{96} e^{-\frac{\phi}{2}}\left(\Gamma_{m}^{n l p} H_{n l p}-9 \Gamma^{n l} H_{m n l}\right) \Gamma^{11} \epsilon+\frac{1}{256} e^{\frac{\phi}{4}}\left(\Gamma_{m}^{n l p q} \tilde{F}_{n l p q}-\frac{20}{3} \Gamma^{n l p} \tilde{F}_{m n l p}\right) \epsilon=0$

This vanishes if we impose:

$$
\tilde{F}_{m n l p}=\frac{1}{6} e^{-\frac{3 \phi}{4}} \sqrt{G_{(7)}} \varepsilon_{m n l p q r s} H^{q r s},
$$

where $G_{(7)}=\operatorname{det}\left(G_{m n}\right)$. After substituting the ansatz (11) (e.g. $G_{m n}=$ $\left.g_{\mathrm{s}}^{-1 / 2} \Delta^{3 / 8} g_{m n}\right)$, we see that all the dependence on the warp factor $\Delta$ disappears and we obtain:

$$
F_{m n l p}+4 A_{[m} H_{n l p]}=\frac{1}{6} g_{\mathrm{s}}^{-1} \sqrt{g_{(7)}} \varepsilon_{m n l p q r s} H_{i j k} g^{q i} g^{r j} g^{s k},
$$

where the metric used is the same $g_{m n}$ as in (11), $g_{(7)}=\operatorname{det}\left(g_{m n}\right)$, and we have used (8).

Thus, the extra conditions for supersymmetry due to the fluxes are (14) and (18), with equation (15) following from the previous two. The fact that (14) and (18) are necessary and sufficient conditions for preservation of the minimal amount of supersymmetry has been shown in [13] in the context 
of 11 dimensional supergravity on a 8-manifold, and the same holds true in our case after trivial dimensional reduction.

It can be checked that the ansatz (11) is indeed a solution to the equations of motion derived from (7) provided the relation (17) is realized. In particular, the equations of motion of the form fields along the directions of the torus would not vanish for an arbitrary configuration of fluxes. The duality relation (17) was derived in this way in a related context in [5] (see also [12]).

The remaining non-trivial equations all reduce to an equation for the warp factor $\Delta$ which reads:

$$
\frac{1}{\sqrt{g_{(7)}}} \partial_{m}\left(\sqrt{g_{(7)}} g^{m n} \partial_{n} \Delta\right)=-\frac{1}{6} H_{m n p} H^{m n p}-\left(2 \pi \sqrt{\alpha^{\prime}}\right)^{5} \rho_{D 2-O 2} .
$$

On the right-hand side of this equation we have included the contribution from localized sources on the torus, that is the number density of orientifold O2-planes and of D2-branes which are possibly present. The integral on the torus of the left-hand side must vanish, and thus the sum of the contributions of the fluxes and the charges must vanish too. This is just the tadpole cancellation condition, since the above equation is also derived from the equations of motion of the 3 -form potential along the 012 directions.

Note that in order for the ansatz (11) to be a solution of the equations of motion in the presence of non-vanishing fluxes, it is sufficient to impose (17). Namely, a solution to the equations of motion can violate (14) and thus be non-supersymmetric.

\section{Generating mass terms for the moduli}

We have seen that the conditions required by supersymmetry on the fluxes $H_{i j l}$ and $F_{i j k l}$ are (14) and (18).

There are two more conditions that the fluxes must satisfy in order to have a consistent string vacuum. The first is the quantization condition which is expressed by:

$$
\frac{1}{2 \pi \alpha^{\prime}} \int_{C} H=2 \pi m, \quad \frac{1}{(2 \pi)^{2} \alpha^{\prime 3 / 2}} \int_{\tilde{C}} F=2 \pi n,
$$

where $C$ and $\tilde{C}$ are respectively three-cycles and four-cycles of $T^{7}$ and we shall always assume $m$ and $n$ to be even to avoid problems with the "halfcycles" discussed in [18] and [19]. 
The second condition is that the total D2-charge induced by the fluxes does not exceed (minus) the orientifold charge. If this condition is satisfied, D2-branes can then be used to make up for the difference in order to cancel the RR tadpole, whereas, if the charge induced by the fluxes exceeded the bound, anti D2-branes would have to be used, thus breaking supersymmetry. In formulas, the condition is:

$$
\frac{1}{2} N_{\text {flux }}=\frac{1}{2} \frac{1}{(2 \pi)^{5} \alpha^{15 / 2}} \int_{T^{7}} H \wedge F \leq 16 .
$$

Equations (20, 21) require that one writes:

$$
\begin{aligned}
H & =\frac{1}{6} \frac{H_{i j k}}{2 \pi \sqrt{\alpha^{\prime}}} d x^{i} \wedge d x^{j} \wedge d x^{k}, \\
F & =\frac{1}{24} \frac{F_{i j k l}}{2 \pi \sqrt{\alpha^{\prime}}} d x^{i} \wedge d x^{j} \wedge d x^{k} \wedge d x^{l},
\end{aligned}
$$

where $x^{i} \sim x^{i}+2 \pi \sqrt{\alpha^{\prime}}$, and we have rescaled the fields in such a way that now $H_{i j k}$ and $F_{i j k l}$ are (even) integers. Equation (21) then requires that those integers obey:

$$
\frac{1}{144} \varepsilon^{i j k l m n p} H_{i j k} F_{l m n p} \leq 32,
$$

where the epsilon tensor is purely numerical.

\subsection{Scalars}

Let us now derive a potential for the candidate moduli. We will start with the massless scalars, that is the dilaton $g_{\mathrm{s}}$, the 28 metric elements $g_{m n}$ and the $7 A_{i}$ scalars.

Equation (18) constrains these moduli, but it is clear that the model always allows for a flat direction characterized by the rescaling $g_{m n} \rightarrow \lambda g_{m n}$ and $g_{\mathrm{s}} \rightarrow \lambda^{1 / 2} g_{\mathrm{s}}$. This modulus will never be stabilized and we shall return to its physical interpretation later on. We can thus write $g_{m n}=g_{\mathrm{s}}^{2} \hat{g}_{m n}$ so that $g_{\mathrm{s}}$ is rescaled away from the relation. It is now clear that we can aim at freezing all of the $\hat{g}_{m n}$ and $A_{i}$ moduli.

The potential for these moduli comes from the kinetic terms for the 3 and 4-form field strengths in (7). Up to an overall numerical factor, it is given by:

$V\left(g_{\mathrm{s}}, \hat{g}_{i j}, A_{i}\right)=\frac{1}{g_{\mathrm{s}}} \sqrt{\hat{g}}\left(\frac{1}{12} H_{i j k} H_{m n p} \hat{g}^{i m} \hat{g}^{j n} \hat{g}^{k p}+\frac{1}{48} \tilde{F}_{i j k l} \tilde{F}_{m n p q} \hat{g}^{i m} \hat{g}^{j n} \hat{g}^{k p} \hat{g}^{l q}\right)$. 
Recall that $\tilde{F}_{i j k l}$ depends on $A_{i}$ as in (8).

We now want to develop this potential around a solution and show that it generically leads to masses for the $\hat{g}_{m n}$ and $A_{i}$ moduli. However, finding the most general solution is a complicated task. We shall bypass this problem by making an ansatz that captures the essential features of the model while allowing for a complete solution. We shall consider a point in moduli space where we know that a solution with integer fluxes exists, namely the square torus $\hat{g}_{i j}=\delta_{i j}, A_{i}=0$ so that we can solve for $F_{i j k l}$ as a function of $H_{i j k}$ :

$$
F_{i j k l}=\frac{1}{6} \varepsilon_{i j k l m n p} H_{m n p}
$$

where, again, $\epsilon_{i j k l m n p}$ is purely numerical and the repeated indices are summed with the square metric $\delta_{i j}$ so they will all be written downstairs.

We can now expand the metric to linear order $\hat{g}_{i j}=\delta_{i j}+h_{i j}$ and rewrite the potential (24) taking into account (25):

$$
\begin{aligned}
g_{\mathrm{s}} V\left(h_{i j}\right) & =\frac{1}{6} H_{i j k} H_{i j k} \\
& +\frac{1}{48} h_{i i} h_{j j} H_{k l m} H_{k l m}-\frac{1}{4} h_{i i} h_{j k} H_{j l m} H_{k l m} \\
& +\frac{1}{4} h_{i j} h_{j k} H_{i l m} H_{k l m}+\frac{1}{2} h_{i j} h_{k l} H_{i k m} H_{j l m} \\
& +\frac{1}{2} h_{i j} A_{k} F_{i k l m} H_{j l m} \\
& +\frac{1}{12} A_{i} A_{i} H_{j k l} H_{j k l}-\frac{1}{4} A_{i} A_{j} H_{i k l} H_{j k l}+\ldots
\end{aligned}
$$

The zeroth order term is actually exactly canceled by the (negative) contribution of the orientifold tension due to the tadpole condition. We note that $\hat{g}_{i j}=\delta_{i j}, A_{i}=0$ is indeed an extremum of the potential. The next step would be to diagonalize the mass matrix derived from the second order term above and count the number of zero eigenvalues.

An alternative way to see how many moduli are frozen is to investigate the space of solutions of the linearized form of eq. (18), after taking into account (25):

$$
h_{i i} H_{m n p}-2\left(h_{i p} H_{m n i}+h_{i m} H_{n p i}+h_{i n} H_{p m i}\right)+2 A_{i} F_{i m n p}=0 .
$$

It can be checked that the quadratic potential (26) is proportional to the square of (27). This is in keeping with the interpretation of the duality equation (18) as coming from the variation of a superpotential. It also implies that all the non-zero eigenvalues of the mass matrix are positive, 
even for non-supersymmetric configurations. Eq. (27) is easier to handle and the problem of counting the number of massless moduli is reduced to that of computing the rank of a $35 \times 35$ matrix with integer coefficients, which can be easily performed by a computer program. A condition for the stabilization of all these moduli is that the system admits only the trivial solution $h_{i j}=0$ and $A_{i}=0$.

Inserting (25) into (23) we also obtain:

$$
\frac{1}{6} H_{i j k} H_{i j k} \leq 32 \text {. }
$$

If we want to saturate the inequality (28), thus allowing for no D2-branes, we have the following three possibilities:

- Eight non-vanishing values for $H_{i j k}$ all equal to 2 ;

- One entry equal to 4 and four equal to 2 ;

- Two non-vanishing values for $H_{i j k}$ both equal to 4 .

Before discussing particular solutions, let us first consider also the massless vectors.

\subsection{Vectors}

The remaining moduli fields would arise as Hodge duals of the 3 dimensional vectors $B_{\mu i}$ and $A_{\mu i j}$. Without fluxes, each one of these fields appears with no interactions whatsoever and can thus be dualized to a massless scalar. In the presence of fluxes, the Chern-Simons (CS) term of the 10 dimensional action will be non-vanishing and this will provide topological mass terms to the 28 vector fields [25].

The correct 3 dimensional CS term is better derived through the reduction of the equations of motion, since in the action (7) the CS term is actually defined up to a total derivative term which is relevant here. After taking that into account, one finds the following 3 dimensional action:

$$
\begin{aligned}
S=\int d^{3} x & {\left[-\frac{1}{4} H_{\mu \nu i} H_{i}^{\mu \nu}-\frac{1}{8} F_{\mu \nu i j} F_{i j}^{\mu \nu}\right.} \\
& \left.-\frac{1}{4} \varepsilon^{\mu \nu \rho}\left(B_{\mu i} F_{\nu \rho j k} H_{i j k}+\frac{1}{4} A_{\mu i j} F_{\nu \rho k l} F_{i j k l}\right)\right],
\end{aligned}
$$

where we have used the relation (25). Note that when the sum is performed over the torus indices all the kinetic terms acquire the canonical normalization and the CS terms have the same numerical prefactor. 
Here we notice that the CS term couples together the 28 vector fields through a matrix which is symmetric in the above fields. It is then possible to diagonalize it by an orthogonal matrix, and every non-vanishing eigenvalue will lead to a topological mass for the relative vector field [25]. Such a topologically massive vector is impossible to dualize to a massless scalar, and hence these fields do not lead to moduli. It is interesting to note that quite generically the eigenvalues of the topological mass matrix break parity. For instance, for a positive eigenvalue there will not necessarily be a negative eigenvalue of the same magnitude.

\subsection{Some examples with $\mathcal{N}=1,2,3,4,5,6$}

Let us now present various configurations of fluxes leading to all possible amounts of supersymmetry allowed by the problem. For each case we will comment on the number of moduli. We consider only cases where the tadpole condition is saturated, that is one of the three cases listed in subsection 4.1. Although a complete classification of the different results obtained by specific choices of the $H_{i j k}$ is beyond the scope of this work, it is interesting to see some of the possible outcomes of the procedure of turning on supersymmetric fluxes.

In the following we will decompose the ten dimensional flat gamma matrices as follows:

$$
\begin{aligned}
\Gamma^{\mu} & =\gamma^{\mu} \otimes \mathbf{1} \otimes \sigma_{1} \\
\Gamma^{i} & =\mathbf{1} \otimes \hat{\gamma}^{i} \otimes \sigma_{2}
\end{aligned}
$$

and consequently, the 32 component spinor $\epsilon$ as a $2 \times 8 \times 2$ spinor $\epsilon=\chi \otimes \eta \otimes \alpha$. The torus gamma matrices are $8 \times 8$ purely imaginary and hermitian.

Supersymmetric configurations (14) can be easily obtained by deriving the equations that preserve a particular spinor $\eta$, that is by writing

$H_{i j k} \hat{\gamma}^{i j k} \eta=0$ for a specifically chosen $\eta$ and choosing $H_{i j k}$ so that the equations are satisfied.

In this section we relabel the coordinate on the torus from $3 \cdots 9$ to $1 \cdots 7$ since there is no possibility of confusion.

$\mathcal{N}=1$

Let us start with a configuration of fluxes which is the most effective in lifting moduli, i.e. where only one modulus is left. An example of $\mathcal{N}=1$ solution freezing the 35 scalars $\hat{g}_{i j}$ and $A_{i}$ is:

$$
H_{123}=H_{127}=H_{136}=H_{235}=H_{236}=H_{257}=H_{347}=H_{357}=2 .
$$


Similarly, one can check that there are no zero eigenvalues to the CS topological mass matrix and consequently there are no moduli arising from the 28 vector fields.

Other cases with $\mathcal{N}=1$ can be found, but many of them will have more than one modulus. One such example is given by:

$$
H_{127}=4, \quad H_{126}=H_{134}=H_{156}=H_{247}=2,
$$

which gives rise to 4 moduli from the scalars, while it gives masses to all the vectors.

$\mathcal{N}=2$

An $\mathcal{N}=2$ configuration can at most freeze all but 2 moduli, since the dilaton/volume has to sit in a supermultiplet, which in turn has to have two bosonic degrees of freedom (for a table of the dimensions of massless supermultiplets in 3 dimensions with various supersymmetries, see 26]). One such configuration is given by:

$$
H_{126}=H_{127}=H_{134}=H_{135}=H_{234}=H_{236}=H_{247}=H_{357}=2 .
$$

The other modulus in the massless supermultiplet with the dilaton/volume arises from the vector fields, since the CS mass matrix has one zero eigenvalue. Note that in this case and all the following ones, the three dimensional graviton multiplet never gives rise to moduli since all its components are purely topological.

Another class of flux configurations that must give $\mathcal{N}=2$ supersymmetries in 3 dimensions is the one obtained by trivial dimensional reduction and T-duality from the minimally supersymmetric configurations of [18]. Under transverse T-duality the $H_{i j k}$ is unaffected and thus we can simply consider solutions where the $H_{i j k}$ fluxes never wrap, say, the 7 th direction of the torus. The minimal number of moduli we expect is thus 8 , since in 4 dimension 18 there were 3 complex moduli together with the graviton multiplet, which upon dimensional reduction gives 2 more massless bosonic degrees of freedom. Indeed, the example:

$$
H_{123}=H_{126}=H_{135}=H_{146}=H_{156}=H_{246}=H_{345}=H_{456}=2,
$$

has 4 massless scalars and 4 massless vectors. 
$\mathcal{N}=3$

More exotic is the case with $\mathcal{N}=3$ supersymmetries. Here the massless multiplets have dimension 4 , so we expect the moduli to come in that multiplicity. An example with only 1 massless supermultiplet is:

$$
H_{127}=H_{134}=H_{156}=-H_{235}=H_{236}=H_{245}=H_{357}=-H_{467}=2,
$$

with the dilaton/volume modulus and 3 massless vectors.

There are many more $\mathcal{N}=3$ cases with more than 1 massless supermultiplet, for instance:

$$
H_{123}=H_{126}=H_{135}=H_{156}=H_{234}=H_{246}=H_{257}=H_{456}=2,
$$

with 3 supermultiplets giving a total of 7 massless scalars and 5 massless vectors.

$\mathcal{N}=4$

$\mathcal{N}=4$ supersymmetries are the generic case when only two fluxes are turned on in a supersymmetric way and saturate the tadpole. In fact, all such cases are equivalent, since the condition $H \eta=0$ has non-zero solutions if and only if the two fluxes have only one direction in common. Furthermore, this will induce a projection which preserves exactly half of the components of the spinor, thus giving $\mathcal{N}=4$. We can thus take:

$$
H_{123}=H_{345}=4,
$$

and find that this class of configurations gives 14 massless moduli and 10 massless vectors, for a total of $6 \mathcal{N}=4$ multiplets.

For $\mathcal{N}=4$ configurations with 8 fluxes turned on, we can find a different number of moduli. For instance, for:

$$
H_{126}=H_{135}=H_{136}=H_{145}=H_{247}=H_{256}=H_{347}=H_{567}=2,
$$

we find 8 massless scalars and 8 massless vectors. Actually, all the other $\mathcal{N}=4$ configurations we have tried have the same set of moduli as one of the two configurations above.

$\mathcal{N}=5$

We have also found $\mathcal{N}=5$ configurations, like:

$$
H_{134}=-H_{156}=H_{236}=-H_{245}=-H_{347}=H_{357}=H_{467}=H_{567}=2,
$$


with 6 massless scalars and 10 massless vectors. All the other $\mathcal{N}=5$ configurations that we have found have the same set of moduli. Note that here the massless supermultiplets are 8-dimensional [26].

$\mathcal{N}=6$

$\mathcal{N}=6$ configurations can be derived from the 4 dimensional $\mathcal{N}=3$ cases of [19]. For instance, for:

$$
H_{123}=H_{126}=H_{135}=H_{156}=H_{234}=H_{246}=H_{345}=H_{456}=2,
$$

we find 16 massless scalars and 16 massless vectors.

It is not possible to have $\mathcal{N}=7$ configurations, since that would imply that $\not H$ has only one non-zero eigenvalue. However, this is impossible because $H$ is a traceless matrix. Thus the minimum amount of non-zero eigenvalues is 2 . The only remaining case, $\mathcal{N}=8$, is the trivial one when the fluxes are not turned on and all the 64 closed string moduli are massless (moreover the tadpole has to be canceled by the addition of 16 D3-branes).

The upshot of this review of particular configurations is that it is fairly easy to come up with a configuration of fluxes for any given amount of supersymmetry which is not ruled out by general arguments. Although for $\mathcal{N}=1,2,3$ it is possible to find configuration with the minimal number of moduli, they do not seem to be generic. Moreover for $\mathcal{N}=4,5,6$ we have not been able to find such configurations, hinting that for higher supersymmetry the low-energy theories deriving from compactifications with fluxes are not generic.

\section{An 11-dimensional perspective}

As we have seen in the previous section, the modulus which is always present is a simultaneous rescaling of the string coupling and of the volume of the torus, in such a way that we can write $g_{i j}=g_{\mathrm{s}}^{2} \delta_{i j}$. This means that the radii of the directions of the torus are given by $R_{i}=g_{\mathrm{s}} \sqrt{\alpha^{\prime}}$. On the other hand, when uplifting a type IIA configuration to M-theory, one gets the same relation for the radius of the 11 th direction, namely $R_{11}=g_{\mathrm{s}} \sqrt{\alpha^{\prime}}$. Thus the correct interpretation is that the modulus is the volume of the 8-dimensional torus in the M-theory picture of the configuration.

In fact, it is possible to do all the analysis in this paper from an Mtheory perspective. One starts by considering M-theory on a $T^{8} / \mathbf{Z}_{2}$ orbifold which is the uplift of the configuration of type IIA theory with the O2planes which we have considered here (see e.g. [27, 28, 29]). Then, upon 
implementing the M2-brane ansatz together with fluxes on $T^{8}$ and requiring supersymmetry, one obtains a condition similar to (15) supplemented by a a relation of self-duality on the torus for the 4-form field strength fluxes. The fluxes now have to saturate a tadpole condition coming from the Euler number of the orbifold which obviously matches the total O2-plane charge in 10-dimensions. The analysis of the potential for the metric moduli and the CS-terms for the vectors goes along very similar lines as in the previous section. The two approaches are clearly exactly equivalent.

We should also consider the order of magnitude of the masses that are generated through the presence of fluxes. It can be shown that all the masses generated by the fluxes, both the ones of the scalars and the topological masses of the vectors, satisfy:

$$
m \sim \frac{\alpha^{\prime}}{R^{3}}
$$

where $R$ is the radius of any direction of the torus, as given by the remaining modulus. Note that this mass can be also expressed as $m \sim\left(g_{\mathrm{s}}^{3} \sqrt{\alpha^{\prime}}\right)^{-1}$. We note now that as long as $R>\sqrt{\alpha^{\prime}}$ (and thus $g_{\mathrm{s}}>1$ ), the masses coming from the fluxes are smaller than any other scale of any other massive object in the theory, be they KK excitations on the torus, string massive states or wrapped D-branes. It is thus consistent, in this regime, to consider a truncated low-energy theory comprising the states which are massive due to the fluxes [18]. Note however that we are not allowed to take $g_{\mathrm{s}}<1$, since this would imply also $R<\sqrt{\alpha^{\prime}}$ and the breakdown of the low-energy description in terms of supergravity (we could however still consider the effective theory of the massless modes).

The fact that $g_{\mathrm{s}}>1$ actually suggests that the best description is the M-theory one. In 11 dimensional language, taking into account the relation $\alpha^{\prime}=l_{p}^{3} / R_{11}$ between the string scale and the 11 dimensional Planck scale, the masses due to the fluxes are given by $m \sim l_{p}^{3} / R^{4}$ and are the smallest scale provided that $R>l_{p}$, which is the regime where the supergravity approach is justified.

\section{Discussion}

To sum up our results, we have explored the locus in moduli space around $g_{\mathrm{s}}^{-2} g_{i j}=\delta_{i j}, A_{i}=B_{\mu i}=A_{\mu i j}=0$, and probed how many of the 64 flat directions are lifted by turning on fluxes on the torus in a supersymmetric way. This requires for instance that the $H_{i j k}$ and $F_{i j k l}$ fluxes are related 
by (25). We have shown that there are $\mathcal{N}=1$ solutions to the equation (14) that freeze all the moduli that can possibly be frozen, that is all but a combination of the dilaton and the volume of the torus. The 35 remaining scalars are fixed through a flux-generated potential that gives them masses when expanding to second order. The 28 vectors do not generate scalars through dualization because the fluxes induce a topological CS mass term for all of them. Moreover we have shown that when picking particular configurations of fluxes, it is possible to produce solutions with all possible amounts of supersymmetry allowed by the set up, and with different numbers of moduli. The case where only one massless supermultiplet is present does not seem to be generic.

The combination of dilaton and volume which remains massless is such that large volume implies strong coupling and vice-versa. This makes it difficult to turn from the purely supergravity point of view that we consider here to a perturbative string theory approach, since we would have to deal either with strong coupling or small volumes. Small volumes would actually imply that the supergravity approximation breaks down, since non-perturbative objects wrapped on the torus would be lighter than the lifted moduli. We are thus driven to consider only the strong coupling, large volume case. Hence from the supergravity perspective we could as well consider that the set up is embedded in 11 dimensions.

We should also point out the issue of whether any modulus in 3 dimensions really remains massless after all perturbative corrections are taken into account. The same issue is relevant for compactifications with fluxes of Mtheory on $\operatorname{Spin}(7)$ manifolds. As discussed in [20] (based on [30]), a massive scalar in a 3 dimensional $\mathcal{N}=1$ theory will receive quantum corrections to its mass at one-loop and non-perturbatively. However when the scalar is massless it can be seen both by standard 3 dimensional arguments (see e.g. [31]) and from the 2 dimensional considerations of [30] that the one-loop correction vanishes. Thus we are left with the non-perturbative corrections, which typically will lead to run-away potentials which lift the moduli but bring the theory to the boundary of the moduli space. The upshot of this is that it is still interesting, and important, to freeze the moduli at a finite value by means of a classical potential, generated by the fluxes in the present case. As for the remaining moduli, the run-away behavior is potentially disturbing, even if it arises only non-perturbatively. See however [22] for a discussion on how $\alpha^{\prime}$ corrections of this type may eventually lead to a stabilization of the remaining moduli.

Our orientifold example is nothing but a tractable model out of a much bigger class of generic models, most simply described as M-theory on $\operatorname{Spin}(7)$ 
manifolds [17, 20]. One could in principle apply the general techniques developed there to our case. In a similar spirit, it should be possible to make contact between our configurations with $\mathcal{N}=2$ supersymmetry and the results about compactifications with fluxes of type II on $G_{2}$ manifolds [11] or M-theory on Calabi-Yau fourfolds [6, 7, 21].

\section{Acknowledgments}

We would like to thank G. Bonelli, B. E. W. Nilsson and A. Zaffaroni for very helpful discussions. R.A. would like to thank the Physics Department at Università di Milano-Bicocca and the Service de Physique Théorique et Mathématique at Université Libre de Bruxelles for their hospitality and for providing a very stimulating environment while this work was completed. This work is partly supported by EU contract HPRN-CT-2000-00122.

\section{References}

[1] J. Polchinski and A. Strominger, "New Vacua for Type II String Theory," Phys. Lett. B 388 (1996) 736 arXiv:hep-th/9510227.

[2] K. Becker and M. Becker, "M-Theory on Eight-Manifolds," Nucl. Phys. B 477 (1996) 155 arXiv:hep-th/9605053.

[3] J. Michelson, "Compactifications of type IIB strings to four dimensions with non-trivial classical potential," Nucl. Phys. B 495 (1997) 127 arXiv:hep-th/9610151.

[4] A. Lukas, B. A. Ovrut and D. Waldram, "Stabilizing dilaton and moduli vacua in string and M-theory cosmology," Nucl. Phys. B 509 (1998) 169 arXiv:hep-th/9611204.

[5] S. W. Hawking and M. M. Taylor-Robinson, "Bulk charges in eleven dimensions," Phys. Rev. D 58 (1998) 025006 arXiv:hep-th/9711042].

[6] S. Gukov, C. Vafa and E. Witten, "CFT's from Calabi-Yau fourfolds," Nucl. Phys. B 584 (2000) 69 [Erratum-ibid. B 608, 477 (2000)] arXiv:hep-th/9906070.

[7] K. Dasgupta, G. Rajesh and S. Sethi, "M theory, orientifolds and Gflux," JHEP 9908 (1999) 023 arXiv:hep-th/9908088. 
[8] C. S. Chan, P. L. Paul and H. Verlinde, "A note on warped string compactification," Nucl. Phys. B 581 (2000) 156 [arXiv:hep-th/0003236].

[9] B. R. Greene, K. Schalm and G. Shiu, "Warped compactifications in M and F theory," Nucl. Phys. B 584 (2000) 480 [arXiv:hep-th/0004103].

[10] P. Mayr, "Stringy world branes and exponential hierarchies," JHEP 0011, 013 (2000) arXiv:hep-th/0006204.

[11] B. S. Acharya and B. Spence, "Flux, supersymmetry and M theory on 7-manifolds," arXiv:hep-th/0007213.

[12] M. Cvetic, H. Lu and C. N. Pope, "Brane resolution through transgression," Nucl. Phys. B 600 (2001) 103 [arXiv:hep-th/0011023].

[13] K. Becker, "A note on compactifications on Spin(7)-holonomy manifolds," JHEP 0105 (2001) 003 arXiv:hep-th/0011114.

[14] G. Curio, A. Klemm, D. Lust and S. Theisen, "On the vacuum structure of type II string compactifications on Calabi-Yau spaces with H-fluxes," Nucl. Phys. B 609 (2001) 3 arXiv:hep-th/0012213.

[15] S. B. Giddings, S. Kachru and J. Polchinski, "Hierarchies from fluxes in string compactifications," arXiv:hep-th/0105097.

[16] G. Curio, A. Klemm, B. Kors and D. Lust, "Fluxes in heterotic and type II string compactifications," Nucl. Phys. B 620 (2002) 237 arXiv:hepth/0106155.

[17] S. Gukov and J. Sparks, "M-theory on $\operatorname{Spin}(7)$ manifolds. I," Nucl. Phys. B 625 (2002) 3 arXiv:hep-th/0109025.

[18] S. Kachru, M. Schulz and S. Trivedi, "Moduli stabilization from fluxes in a simple IIB orientifold," arXiv:hep-th/0201028.

[19] A. R. Frey and J. Polchinski, "N = 3 warped compactifications," arXiv:hep-th/0201029.

[20] B. Acharya, X. de la Ossa and S. Gukov, "G-flux, Supersymmetry and Spin(7) manifolds," arXiv:hep-th/0201227.

[21] S. Gukov and M. Haack, "IIA string theory on Calabi-Yau fourfolds with background fluxes," arXiv:hep-th/0203267. 
[22] K. Becker, M. Becker, M. Haack and J. Louis, "Supersymmetry breaking and alpha' corrections to flux induced potentials," arXiv:hepth/0204254.

[23] M. Mueller and E. Witten, "Twisting Toroidally Compactified Heterotic Strings With Enlarged Symmetry Groups," Phys. Lett. B 182 (1986) 28;

J. A. Harvey, G. W. Moore and C. Vafa, "Quasicrystalline Compactification," Nucl. Phys. B 304 (1988), 269;

M. Dine and E. Silverstein, "New M-theory backgrounds with frozen moduli," arXiv:hep-th/9712166;

A. Dabholkar and J. A. Harvey, "String islands," JHEP 9902 (1999) 006 arXiv:hep-th/9809122.

[24] M. Huq and M. A. Namazie, "Kaluza-Klein Supergravity In TenDimensions," Class. Quant. Grav. 2 (1985) 293 [Erratum-ibid. 2 (1985) 597];

F. Giani and M. Pernici, "N=2 Supergravity In Ten-Dimensions," Phys. Rev. D 30 (1984) 325;

I. C. Campbell and P. C. West, "N=2 D = 10 Nonchiral Supergravity And Its Spontaneous Compactification," Nucl. Phys. B 243 (1984) 112.

[25] S. Deser, R. Jackiw and S. Templeton, "Topologically Massive Gauge Theories," Annals Phys. 140 (1982) 372 [Erratum-ibid. 185 (1982) 406.1988 APNYA,281,409].

S. Deser, R. Jackiw and S. Templeton, "Three-Dimensional Massive Gauge Theories," Phys. Rev. Lett. 48 (1982) 975.

[26] B. de Wit, A. K. Tollsten and H. Nicolai, "Locally supersymmetric D = 3 nonlinear sigma models," Nucl. Phys. B 392 (1993) 3 arXiv:hepth/9208074.

[27] A. Sen, "Orbifolds of M-Theory and String Theory," Mod. Phys. Lett. A 11 (1996) 1339 arXiv:hep-th/9603113.

[28] S. Sethi, "A relation between $N=8$ gauge theories in three dimensions," JHEP 9811 (1998) 003 arXiv:hep-th/9809162.

[29] A. Hanany and B. Kol, "On orientifolds, discrete torsion, branes and M theory," JHEP 0006 (2000) 013 [arXiv:hep-th/0003025]. 
[30] M. A. Shifman, A. I. Vainshtein and M. B. Voloshin, "Anomaly and quantum corrections to solitons in two-dimensional theories with minimal supersymmetry," Phys. Rev. D 59 (1999) 045016 arXiv:hepth/9810068.

[31] S. J. Gates, M. T. Grisaru, M. Rocek and W. Siegel, "Superspace, Or One Thousand And One Lessons In Supersymmetry," Front. Phys. 58 (1983) 1 arXiv:hep-th/0108200. 